\title{
Concordância entre Diagnósticos de Doença Renal Crônica em pessoas vivendo com HIV
}

\section{Agreement between chronic kidney disease diagnosis in people living with HIV}

Priscila Silva Pontes ${ }^{1}$, Christefany Régia Braz Costa², Elizabete Santos Melo3, Layze Braz de Oliveira ${ }^{4}$, Elucir Gir ${ }^{5}$, Antonio Ruffino-Netto ${ }^{6}$, Renata Karina Reis ${ }^{7}$

1. ORCID: http://orcid.org/0000-0002-1318-8431. Enfermeira. Mestre. Universidade de São Paulo, Escola de Enfermagem de Ribeirão Preto, Ribeirão Preto, SP, Brasil. E-mail: 2. priscilapontes@usp.br.

2. ORCID: http://orcid.org/0000-0001-6124-8243. Enfermeira. Mestre. Universidade de São Paulo, Escola de Enfermagem de Ribeirão Preto, Ribeirão Preto, SP, Brasil. E-mail: christefany.costa@usp.br

3. ORCID: https://orcid.org/0000-0002-7322-9370. Enfermeira. Doutora. Universidade Paulista - UNIP, São José do Rio Preto, SP, Brasil Filiação (instituição, cidade, estado e país). E-mail: enfaelizabetemelo@gmail.com

4. ORCID: https://orcid.org/0000-0002-9542-1451. Enfermeira. Mestre. Universidade de São Paulo, Escola de Enfermagem de Ribeirão Preto, Ribeirão Preto, SP, Brasil. E-mail: layzebraz@gmail.com

5. ORCID: https://orcid.org/ 0000-0002-3757-4900. Enfermeira. Pós-Doutora. Universidade de São Paulo, Escola de Enfermagem de Ribeirão Preto, Ribeirão Preto, SP, Brasil. E-mail: egir@eerp.usp.br.

6. ORCID: http://orcid.org/0000-0001-9770-4896. Médico. Pós-Doutor. Universidade de São Paulo, Faculdade de Medicina de Ribeirão Preto, Ribeirão Preto, SP, Brasil. E-mail: aruffino@fmrp.usp.br

7. ORCID: http://orcid.org/0000-0002-0681-4721. Enfermeira. Pós-Doutora. Universidade de São Paulo, Escola de Enfermagem de Ribeirão Preto, Ribeirão Preto, SP, Brasil. E-mail: rkreis@eerp.usp.br

CONTATO: Nome do autor correspondente: Priscila Silva Pontes | Endereço: Condomínio Parque Jatiúca Q.e BI. 06 Ap. 04 CEP: 57035-610. Telefone: (82) 99608-0112 . E-mail: priscilapontes@usp.br 
RESUMO Analisar concordância entre diagnósticos de Doença Renal Crônica em prontuários clínicos e pelo critério da Sociedade Brasileira de Nefrologia em Pessoas vivendo com HIV. Trata-se de um estudo descritivo de concordância de diagnósticos, através de levantamento de prontuários. A população do estudo foi de 258 pessoas vivendo com HIV atendidas em um serviço de atendimento especializado localizado em um interior de São Paulo, no ano de 2017. Para cálculo da concordância foi utilizado o índice Kappa, copositividade e conegatividade. Encontramos 6,5\% pacientes com Doença Renal Crônica pelo critério da Sociedade Brasileira de Nefrologia; e 5,42\% pelo diagnóstico médico. O índice Kappa foi de 0,55; a copositividade de 52,9\% e conegatividade de $97,9 \%$. Identificou-se uma concordância dos diagnósticos moderada, copositividade baixa e alta conegatividade. O que aponta uma importância de investigações precisas dos diagnósticos pautados em mais de um critério avaliativo.

DESCRITORES: Registros Médicos. Insuficiência Renal Crônica. Síndrome de Imunodeficiência Adquirida.

ABSTRACT To analyze the agreement between Chronic Kidney Disease diagnoses in clinical records and by the criteria of the Brazilian Society of Nephrology in People Living with HIV. This is a descriptive study of diagnostic agreement, through medical records survey. The study population consisted of 258 people living with HIV attended at a specialized care service located in some interior of São Paulo State, in 2017. To calculate the agreement, the Kappa index, copositivity and connectivity was used. We found $6.5 \%$ patients with Chronic Kidney Disease according to the criteria of the Brazilian Society of Nephrology; and $5.42 \%$ by medical diagnosis. The Kappa index was 0.55 ; the copositivity of $52.9 \%$ and connectivity of $97.9 \%$. A moderate diagnoses concordance, low copositivity and high connectivity was identified. This indicates the importance of accurate investigations of diagnoses based on more than one evaluative criterion.

DESCRIPTORS: Medical Records. Chronic Kidney Failure. Acquired Immunodeficiency Syndrome. 


\section{INTRODUÇÃO}

Os avanços no campo terapêutico definiram um novo panorama na perspectiva de vida das pessoas vivendo com HIV/aids (PVHIV) ${ }^{1}$. Após o sucesso dos antirretrovirais (ARV) para o controle da replicação viral, esta população tem alcançado melhor qualidade de vida e maior longevidade ${ }^{2}$. Neste cenário, a infecção do vírus do HIV tornou-se uma condição crônica ${ }^{3}$. No entanto, mudanças no perfil da saúde desta população justifica os elevados riscos de desenvolver comorbidades, principalmente em decorrência dos aspectos fisiopatológicos, hábitos de vida e medicamentos ${ }^{4}$. Portanto, a assistência à saúde destes indivíduos enfrenta desafios clínicos, gerenciais e econômicos ${ }^{5}$.

Dentre as comorbidades, a doença renal crônica (DRC) apresenta uma alta prevalência em PVHIV quando comparado à população geral ${ }^{4,6,7}$. Sua variação é atribuída à heterogeneidade genética, inconsistência no acesso à saúde e diferentes critérios de definição para $\mathrm{DRC}^{8,9}$. Esta divergência também varia de forma geográfica e populacional, correspondendo entre $2 \%$ e $38 \% \%^{5}$. A Sociedade Brasileira de Nefrologia (SBN) define os critérios para DRC, os quais são utilizados pelos profissionais de saúde em âmbito am-

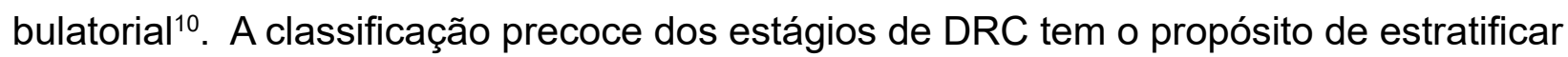
os possíveis riscos, além de ser importante para a decisão do tratamento adequado ${ }^{10}$.

Ademais, evidências apontam um desconhecimento dos profissionais de saúde acerca do manejo desta população, além da fragilidade no fluxo de atendimento dos serviços de saúde ${ }^{11,12}$. Nesse contexto, ações gerenciais e registros multiprofissionais permitem a continuidade da assistência e o estabelecimento de condutas ${ }^{13,14}$. Isto representa um aspecto ético/legal a todos os envolvidos no processo do cuidado ${ }^{13,14}$.

Frente ao exposto, a utilização de dados secundários implica tomar conhecimento da qualidade dos mesmos, tais como disponibilidade, completude e acuidade para diagnósticos precoces e assertivos. Assim, o objetivo deste estudo foi analisar a concordância entre diagnósticos de DRC em prontuários clínicos e pelo critério da SBN, em PVHIV.

\section{MÉTODO}

Trata-se de um estudo descritivo de concordância de diagnósticos, através de levantamento de prontuários. A população de referência foram pessoas vivendo com HIV/aids e a população de estudo, as PVHIV atendidas em um Serviço de Atendimento Especializado (SAE) no interior do estado de São Paulo. O período de coleta de dados correspondeu de fevereiro a maio de 2018.

Foram incluídos no estudo PVHIV em acompanhamento clínico-ambulatorial no SAE; maior ou igual a 18 anos; em uso de terapia antirretroviral (TARV) há mais de 6 meses; e que possuíam mais de um resultado de exame de creatinina sérica. Foram 
excluídas pessoas com diagnóstico de doença renal aguda ou crônica previamente ao diagnóstico de HIV; em hemodiálise antes de iniciar a TARV e ter abandonado o tratamento com antirretrovirais.

A população elegível identificada no serviço escolhido era composta por 776 PVHIV em acompanhamento ambulatorial no ano de 2017. Para o cálculo do tamanho amostral foi utilizado a seguinte fórmula: $n=\frac{Z \alpha^{2} \cdot(P, Q)}{d^{2}} n=\frac{Z \alpha^{2} \cdot(P, Q)}{d^{2}}$, cálculo para estudos descritivos, onde $n n$ (tamanho amostral), $Z Z$ (variável reduzida), $\alpha=5 \% \alpha=5 \%, P=50 \% P=50 \%$, $d($ nível de precisão $)=5 \% d$ (nível de precisão $)=5 \%$. Após correção para uma população finita, o tamanho amostral foi de 258. Tratou-se de amostragem de conveniência, isto é, foi selecionado o primeiro prontuário que satisfez os critérios de inclusão.

Para cada um deles levantou-se as informações que permitissem a identificação do diagnóstico de DRC. Foram levantados nos prontuários clínicos as informações que embasam o diagnóstico de DRC segundo um dos critérios preconizados pela SBN. Analisou-se as três últimas estimativas das Taxas de Filtração Glomerular (eTFG).

De acordo com a SBN é portador de DRC qualquer indivíduo que, independente da causa, apresente TFG $<60 \mathrm{ml} / \mathrm{min} / 1,73 \mathrm{~m}^{2}$ pelo menos três meses consecutivos. Nos casos de pacientes com TFG $\geq 60 \mathrm{ml} / \mathrm{mim} / 1,73 \mathrm{~m}^{2}$, considerar DRC se associada a pelo menos um marcador de dano renal parenquimatoso ou alteração no exame de imagem ${ }^{10}$.

O critério de DRC utilizado para este estudo foi: apresentar duas ou mais eTFG $<60$ $\mathrm{mL} / \mathrm{min} / 1,73 \mathrm{~m}^{2}$ estimada pela Equação do grupo Chronic Kidney Disease Epidemiology Collaboration (CKD-EPI) em um período de três meses ou mais, portanto, apenas um dos critérios da SBN foi contemplado. Quanto ao levantamento do diagnóstico médico de DRC, esta foi realizada diretamente dos prontuários clínicos dos pacientes.

Para uma descrição geral da população estudada, levantamos as informações contidas nos prontuários clínicos, quais sejam: sociodemográficas= sexo (masculino e feminino), idade ( $<40$ anos, 40-60 anos e $>60$ anos) e cor de pele (amarela, branca, parda, preta, não declara), escolaridade ( $\leq 8$ anos e $>8$ anos de estudo), comportamental= tabagismo (sim e não), clínicos= Diabetes Mellitus- DM (sim e não), Hipertensão Arterial Sistêmica- HAS (sim e não), Doença Cardiovascular- DCV (sim e não), dislipidemia (sim e não), litíase renal (sim e não), infecção do trato urinário de repetição- ITU (sim e não), uso de antirretroviral nefrotóxico- Tenofovir Disoproxil Fumarato (TDF) (sim e não), os três últimos exames laboratoriais de creatinina sérica.

As variáveis: creatinina sérica, idade, sexo e cor de pele foram inseridas na calculadora Nefrocalc 2.0 da SBN para se obter a eTFG pela equação CKD-EPI ${ }^{15}$. Inicialmente converteu-se a creatinina sérica convencional em Creatinina de Espectrometria de Massa de Diluição Isotópica (IDMS), considerada padrão de referência ${ }^{15}$. 
Neste estudo foram considerados os estágios de DRC: $3 A$ (eTFG: $45-59 \mathrm{~mL} / \mathrm{min} / 1,73$ m²); 3B (eTFG: $30-44$ mL/min/1,73 m²); 4 (eTFG:15-29 mL/min/1,73 m²) e 5 (eTFG < $15 \mathrm{~mL} /$ $\left.\mathrm{min} / 1,73 \mathrm{~m}^{2}\right)^{16}$ pelo motivo de falha na disponibilidade dos exames de proteinúria de 24 horas e/ou relação Albuminúruia/creatininúria (RAC) nos prontuários clínicos. Os estágios de DRC: 1 e 2 não foram contemplados por necessitar de análises da proteinúria de 24 horas.

Os dados foram processados e analisados por meio do software Statistical Package for Social Science (SPSS), versão 25.0. Utilizou-se estatística descritiva (frequência relativa e absoluta, média, desvio padrão, mínima e máxima). Os cálculos para a concordância (índice Kappa, copositividade e conegatividade) entre os dois processos de diagnóstico, foram calculados em tabela de contingência ou tabela $2 \times 2$ (Tabela 1) ${ }^{17}$.

Tabela 1. Tabela de contingência $2 \times 2$ utilizada para análise de concordância.

\begin{tabular}{cccc}
\hline Teste & \multicolumn{2}{c}{ Padrão ouro } & Total \\
\hline Positivo & Positivo & Negativo & \\
Negativo & $\mathrm{A}$ & $\mathrm{B}$ & $\mathrm{a}+\mathrm{b}$ \\
Total & $\mathrm{C}$ & $\mathrm{D}$ & $\mathrm{c}+\mathrm{d}$ \\
& $\mathrm{a}+\mathrm{c}$ & $\mathrm{b}+\mathrm{d}$ & $\mathrm{N}$ \\
\hline
\end{tabular}

Fonte: Bastos (2016) ${ }^{17}$.

A partir dos critérios propostos por Landis e Koch, o coeficiente Kappa segue a seguinte escala: sem concordância $(<0)$; concordância pobre $(0$ a 0,19$)$; concordância razoável $(0,20$ a 0,39$)$; concordância moderada $(0,40$ a 0,59$)$; concordância substancial $(0,60$ a 0,79$)$, e excelente concordância $(0,80 \text { a } 1,00)^{18}$. A copositividade: $a /(a+c)$ é definida como a capacidade de um teste detectar os indivíduos verdadeiramente positivos (doentes) e conegatividade: $d /(b+d)$, identifica corretamente os indivíduos sadios.

O estudo foi conduzido de acordo com as normas legais e éticas da Resolução 466/2012. O mesmo foi aprovado pelo Comitê de Ética em Pesquisa da Escola de Enfermagem de Ribeirão Preto da Universidade de São Paulo sob o protocolo $n^{\circ} 2439.373$. Sob n ${ }^{\circ}$ CAAE: 79881717.8 .0000 .5393 aprovado em 14 de dezembro de 2017.

\section{RESULTADOS}

Dos 258 prontuários analisados inicialmente, encontramos 17 (6,5\%) pacientes com DRC pelo critério da SBN e 14 (5,42\%) pelo diagnóstico médico registrados em prontuários clínicos.

A Tabela 2 apresenta as características sociodemográficas e comportamental dos 258 participantes. Destes, a maioria era do sexo masculino (64,7\%), tinham entre 40 a 60 anos $(50 \%)$, declaravam cor de pele branca ( $43 \%)$, apresentavam uma baixa escolaridade ( $\leq 8$ anos de estudo) $(34,9 \%)$ e eram não tabagistas $(67,4 \%)$. 
Tabela 2. Distribuição de frequência absoluta (n) e relativa (\%) das variáveis sociodemográficas e comportamental das pessoas vivendo com HIV/aids (N=258), Ribeirão Preto-SP, 2018.

\begin{tabular}{lcc}
\hline & Variáveis & $\mathbf{n}(\%)$ \\
\hline Sexo & Masculino & $167(64,7)$ \\
Idade (em anos) & Feminino & $91(35,3)$ \\
& $<40$ & $92(35,6)$ \\
Cor de pele & $40-60$ & $129(50,0)$ \\
& $\geq 60$ & $37(14,3)$ \\
& & \\
& Amarela & $0(0)$ \\
Escolaridade & Branca & $111(43,0)$ \\
& Parda & $72(27,9)$ \\
Tabagismo & Preta & $17(6,6)$ \\
& Não declara & $58(22,5)$ \\
Total & $\leq 8$ anos & $90(34,9)$ \\
& $>8$ anos & $41(15,9)$ \\
& Não declara & $127(49,2)$ \\
& & $84(32,6)$ \\
& Sim & $174(67,4)$ \\
\hline
\end{tabular}

Fonte: Elaborado pelos autores (2020)

A distribuição das variáveis clínicas é apresentada na Tabela 3. Nesta observa-se que $7,0 \%$ diabéticos, $21,3 \%$ hipertensos, $6,2 \%$ tinham alguma doença cardiovascular, 7,4\% litíase renal e 7,0\% Infecção do Trato Urinário de repetição; 79,5\% dislipidemia; e 83,7\% faziam uso de antirretroviral nefrotóxico (TDF).

Tabela 3. Distribuição de frequência absoluta ( $\mathrm{n}$ ) e relativa (\%) de pessoas vivendo com HIV/ aids segundo e variáveis clínicas (N=258), Ribeirão Preto- SP, 2018.

\begin{tabular}{ccc}
\multicolumn{2}{c}{ Variáveis } & $\mathrm{n}(\%)$ \\
\hline Diabetes Mellitus & Sim & $18(07,0)$ \\
& Não & $240(93,0)$ \\
$\begin{array}{c}\text { Hipertensão Arterial } \\
\text { Sistêmica }\end{array}$ & Sim & \\
Noença cardiovascular & Sim & $203(21,3)$ \\
& Não & $16(6,2)$ \\
Dislipidemia & & $231(89,5)$
\end{tabular}




\begin{tabular}{ccc} 
& Sim & $205(79,5)$ \\
Litíase renal & Não & $52(20,2)$ \\
& Sim & $19(7,4)$ \\
$\begin{array}{c}\text { Infecção do trato urinário } \\
\text { de repetição }\end{array}$ & Não & $239(92,6)$ \\
$\begin{array}{c}\text { Uso de Tenofovir } \\
\text { Disoproxil Fumarato }\end{array}$ & Não & $18(07,0)$ \\
& Sim & $240(93,0)$ \\
Total & Não & $216(83,7)$ \\
& & $42(16,3)$ \\
\hline Fonte: Elaborado pelos autores (2020) & & $258(100,0)$
\end{tabular}

Fonte: Elaborado pelos autores (2020)

Na Tabela 4 apresentamos a concordância encontrada entre os diagnósticos feitos segundo um dos critérios preconizados pela SBN e os diagnósticos realizados pelos médicos registrados em prontuários clínicos. Nesta, observamos que o índice Kappa para a concordância foi de 0,55; a copositividade: $9 / 17=0,529$, ou seja, 52,9\% e conegatividade: $236 / 241=0,979$, ou seja, $97,9 \%$.

Tabela 4. Concordância entre os diagnósticos de DRC (diagnóstico médico em prontuário clínico $x$ diagnóstico por critério da SBN), índice Kappa, copositividade e conegatividade, Ribeirão Preto- SP, 2018.

\begin{tabular}{llccc}
\hline & & \multicolumn{3}{c}{ Diagnóstico de DRC pelo critério da SBN } \\
\cline { 3 - 5 } & & Com DRC & Sem DRC & TOTAL \\
\hline \multirow{2}{*}{ Diagnóstico médico } & Com DRC & 09 & 05 & 14 \\
& Sem DRC & 08 & 236 & 244 \\
& TOTAL & 17 & 241 & 258 \\
\hline
\end{tabular}

Fonte: Elaborado pelos autores (2020)

Nota: Kappa:0,55; Copositividade:52,9\%; Conegatividade:97,9\%.

\section{DISCUSSÃO}

No presente estudo, identificou-se uma concordância moderada entre os diagnósticos de DRC por um dos critérios da SBN e o diagnóstico registrado pelo médico em prontuários. Observamos uma baixa copositividade entre os diagnósticos $(52,9 \%)$. Já a conegatividade foi elevada $(97,9 \%)$.

Visto que a concordância é moderada, este fato nos remete a algumas reflexões sobre a prevalência de DRC em PVHIV. Neste estudo encontramos uma prevalência de 6,5\% 
de DRC segundo o critério da SBN e 5,42\% pelo critério de diagnóstico médico. Outros pesquisadores que usaram o critério de eTFG $<60 \mathrm{~mL} / \mathrm{min} / 1,73 \mathrm{~m}^{2}$, sugeriram uma prevalência entre $3 \%$ a $13,4 \%$, 19-22. Por outro lado, estudos que analisaram eTFG e proteinúria de 24 horas evidenciaram uma prevalência global de $11,7 \%$ a 18,3\% $\%^{23,24}$.

Estes dados confirmam que a utilização de mais de um critério avaliativo da função renal evidencia uma maior prevalência de DRC em PVHIV. Sua alta ocorrência é um desafio para a saúde pública em países com alta prevalência de HIV em que o acesso aos cuidados é insuficiente e/ou ineficiente ${ }^{8}$. Tal situação pode acarretar pressões econômicas refletindo o mal financiamento a altos custos na saúde ${ }^{8}$.

Vale frisar que a DRC é uma condição muitas vezes assintomática e por isso seu prognóstico permanece ruim. O conhecimento dos fatores de riscos oportuniza uma integração de cuidados a doenças crônicas em PVHIV de uma maneira racional proporcionando melhores interveções para redução de danos ${ }^{25}$.

Em vista do problema de saúde pública, é pertinente levantar os seguintes questionamentos: Quais os critérios para o diagnóstico de DRC foram utilizados pelos médicos do serviço? Houve uma devida valorização nos exames de função renal? Além de indagar sobre os possíveis casos que não foram diagnósticados oportunamente (em estágios iniciais da doença), impossibilitando assim tratamentos específicos.

Seria ainda oportuno se pensar que, se nos critérios de definição de DRC tivéssemos utilizado também a dosagem de proteinúria de 24 horas, os casos diagnosticados levaria a uma prevalência maior que $6,5 \%$, ou seja, aumentaria a discordância entre os diagnósticos da pesquisa e do médico. Supomos assim que, o número de PVHIV com DRC possivelmente seja superior ao atualmente detectado, e consequentemente, muitos estão sem o possível diagnóstico e tratamento.

Destaca-se ainda a importância da qualidade do diagnóstico, o qual é comumente realizado após achados de testes de rastreio, ou quando os sintomas se tornam graves $^{26}$. No entanto, para um rastreio eficaz, faz-se necessário a sensibilização dos profissionais de saúde quanto as necessidades desses pacientes ${ }^{27}$. Além de garantir a oferta de apoio diagnóstico e terapêutico adequado para pacientes com DRC e seus fatores de risco ${ }^{28}$.

Nesse contexto, a utilização dos protocolos assistenciais é apropriada, visto que estabelecem critérios diagnósticos para doença e/ou para o agravo à saúde. Sua contribuição é importante para um melhor acompanhamento dos resultados terapêuticos, além de favorecer a padronização do cuidado em saúde ${ }^{29}$. Aliado aos protocolos, o registro no prontuário coopera para o cuidado integral direcionando a assistência e reafirmando a identidade profissional ${ }^{29}$. 
Assim, além dos aspectos anteriormente citados, deve-se refletir quanto a: qualidade de diagnóstico realizado nos serviços de saúde (e portanto, na formação de recursos humanos para a saúde); no planejamento das atividades; nos aspectos de comunicação verbal e não verbal entre a equipe multiprofissional e intersetorial; na organização dos serviços de saúde; na vigilância epidemiológica e consequentemente nos aspectos operacionais e econômicos envolvidos em todo o processo.

A limitação deste estudo é referente à falta de mais de um resultado de exames laboratoriais de proteinúria de 24 horas e/ou RAC nos prontuários clínicos dos pacientes, o que impossibilitou a identificação de pessoas com DRC em estágios iniciais da doença (1 e 2), subestimando assim o quantitativo de PVHIV com esta patologia. Sugere-se que avaliação periódica da função renal seja realizada segundo o Protocolo Clínico de Diretrizes terapêuticas (PCDT) para PVHIV e pelas diretrizes da SBN.

Tais limitações não inviabilizaram a realização do estudo. Assim como este, outros estudos internacionais apontaram inconveniências semelhantes quanto ao acesso aos exames de proteinúria. Tais estudiosos utilizaram a mesma definição adotada neste estudo 8,14,21.

\section{CONCLUSÃO}

Julgamos que no presente estudo, a concordância dos diagnósticos moderada, copositividade baixa e alta conegatividade apontam a importância de uma investigação precisa dos diagnósticos pautados em mais de um critério avaliativo. Tais aspectos mostram que é possível a realização de pesquisas nos serviços de saúde, desde que estejamos atentos em conhecer a acuidade das informações registradas nos referidos serviços.

Além disso, vale ressaltar a relevância da realização de novos estudos que analisem a concordância entre diagnósticos preconizados por diretrizes de sociedades nacionais e/ ou internacionais e o diagnóstico médico em prontuários clínicos nos serviços de saúde, com o intuito de verificação de precisão diagnóstica e eventuais tratamentos oportunos. Com tais resultados, é possível recomendar a secretarias de saúde municipais e estaduais, além dos próprios serviços de saúde, a realização de capacitações, atualizações e apresentação dos indicadores de saúde aos profissionais de saúde.

O presente trabalho foi realizado com apoio da Coordenação de Aperfeiçoamento de Pessoal de Nível Superior - Brasil (CAPES), número do processo: 001.

\section{REFERÊNCIAS}

1.Tancredi MV, Waldman EA.Survival of AIDS patients in Sao Paulo-Brazil in the pre- and post-HAART eras: a cohort study. BMC Infect Dis. 2014;14:599. https://doi.org/doi: 10.1186/s12879-014-0599-8.

2.World Health Organization. "Chronic conditions: The global burden" e "Chronic disease". 2017 [citado em 2019 ago 23]. Disponível em: http://www.who.int/chronic_condition/burden/en/index.html 
3.Brasil. Ministério da Saúde. Departamento de Vigilância, Prevenção e Controle das Infecções Sexualmente Transmissíveis, do HIVIAids e das Hepatites Virais. Protocolo Clínico e Diretrizes Terapêuticas para o Manejo da Infecção pelo HIV em adultos. 2018 [citado em 2019 ago 01]. Disponível em: http://www.aids. gov.br/pt-br/pub/2013/protocolo-clinico-e-diretrizes-terapeuticas-para-manejo-da-infeccao-pelo-hiv-em-adultos.

4.Mocroft A, Lundgren JD, Ross M, Fux CA, Reiss P, Moranne O, et al. Cumulative and current exposure to potentially nephrotoxic. antiretrovirals and development of chronic kidney disease in HIV- positive individuals with a normal baseline estimated glomerular filtration rate: a prospective internacional cohort study. Lancet HIV; 2015; 3(1):23-32. http://dx.doi.org/10.1016/ S2352-3018(15)00211-8

5.Ekrikpo EU, Kengne AP, Bello AK, Effa EE, Noubiap JJ, Salako BL, et al. Chronic kidney disease in the global adult HIVinfected population: A systematic review and meta-analysis. PLoS ONE. 2018;13(4):e0195443. https://doi.org/10.1371/journal. pone.0195443

6.Ryom L, Lundgren JD, Ross M, Kirk O, Law M, Morlat P, et al. Renal impairment and cardiovascular disease in HIV-positive individuals: The D:A:D study. J Infect Dis; J Infect Dis. 2016;214(8):1212-20. http:// dx.doi.org/10.1093/infdis/jiw342.

7.Kopp JB. Chronic Kidney Disease in the Aging Human Immunodeficiency Virus-Infected Population. J Infect Dis. 2017; 216(6):619-621. http://dx.doi.org/10.1093/infdis/jix205.

8.Ekrikpo UE, Kengne AP, Akpan EE, Effa EE, Bello AK, Ekott JU, et al. Prevalence and correlates of chronic kidney disease (CKD) among ART-naive HIV patients in the Niger-Delta region of Nigeria. Medicine (Baltimore). 2018;97(16):e0380. http://dx.doi.org/10.1097/MD.0000000000010380.

9.Rosenberg AZ, Naicker S, Winkler CA, Kopp JB. HIV-associated nephropathies: epidemiology, pathology, mechanisms and treatment. Nat Rev Nephrol. 2015; 11(3):150-60. http://dx.doi: 10.1038/nrneph.2015.9

10.Brasil. Ministério da Saúde. Secretaria de Atenção à Saúde. Departamento de Atenção Especializada e Temática. Diretrizes Clínicas para o Cuidado ao paciente com Doença Renal Crônica- DRC no Sistema Único de Saúde. 2014 [citado em 2019 ago 15]. Disponível em: http://bvsms.saude.gov.br/bvs/publicacoes/diretrizes_clinicas_cuidado_paciente_renal.pdf.

11.Zambenedetti G, Silva RAN. Descentralização da atenção em HIV-Aids para a atenção básica: tensões e potencialidades. Physis [Internet]. 2016 [acesso em 2019 ago 15]; 26(3): 785-806. Disponível em: http:// dx.doi.org/10.1590/s0103-73312016000300005.

12.Cordeiro HP, Silva RA, Tavares L F, Bichara CNC. Desenvolvimento de um aplicativo para atendimento médico de pessoas que vivem com HIVIAIDS. Revista Eletrônica Acervo Saúde [Internet]. 2019 [acesso em 2019 ago 15]; 22: e139. Disponível em: https://doi.org/10.25248/reas.e139.201.

13.Figueiredo T, Da Silva PLN, Guimarães CF, Oliveira MKS, Alves ECS. Avaliação dos Registros de Enfermagem de Pacientes Internados na Clínica Médica de um Hospital Universitário do Norte do Estado de Minas Gerais. Rev. pesqui. cuid. fundam.[Internet]; 2019 [acesso em 2019 ago 20];11(2):390-396. Disponível em: http://dx.doi.org/10.9789/2175-5361.2019.v11i2.390-396

14.Alves KY, Oliveira PT, Chiavone FB, Barbosa ML, Saraiva CO, Martins CC, et al. Identificação do paciente nos registros dos profissionais de saúde. Acta Paul Enferm. 2018;31(1):79-86. http://dx.doi. org/10.1590/1982-0194201800012

15.Sociedade Brasileira de Nefrologia. Passo a passo para a implantação da estimativa da taxa de filtração glomerular (eTFG). 2.ed. [Internet]. 2015 [citado em 20 feb 2019]. Disponível em: www.sbpc.org.br/upload/ conteudo/padronizacao_eTFG_4nov2015.pdf

16.Levin A, Stevens PE, Bilous RW, Coresh J, De Francisco ALM, De Jong PE, et al. Kidney disease: Improving global outcomes (KDIGO) CKD work group. KDIGO 2012 clinical practice guideline for the evaluation and management of chronic kidney disease. Kidney International Supplements. 2013; 3(1):1-150. https://doi.org/10.1038/kisup.2012.73.

17.Bastos RR. Medidas de variação inter- observador: reprodutibilidade no contexto clínico- Curso continuado de bioestatística e epidemiologia. HU revista. 2016; 30(2-3): 79-81. http://www.ufjf.br/hurevista/ files/2016/11/extracted_72-8-PB-9.pdf 
18.Landis JR, Koch GG. The Measurement of Observer Agreement for Categorical Data. International Biometric Society. 1977;33(1):159-74.

19.Alves TP, Wu P, T.Ikizler TA, Sterling TR, Stinnette SE, Rebeiro PF et a. Chronic kidney disease at presentation is not an independent risk factor for AIDS-defining events or death in HIV-infected persons. Clinical Nephrology, 2013;79(2):93-100. https://doi.org/10.5414/CN107390

20.Costa ES, Oliveira DEP, Vieira FS, Sousa GC, Moura MES. Avaliação da função renal de pacientes com vírus da imunodeficiência humana. Rev Rene. 2017; 18(2):220-6. https://doi. org/10.15253/2175-6783.2017000200011

21.Woolnough EL, Hoy JF, Cheng AC, Walker RG, Chrysostomou A, Woolley I. Predictors of chronic kidney disease and utility of risk prediction scores in HIV positive individuals. AIDS. 2018;32(13):1829-35. https:// doi.org/10.1097/QAD.0000000000001901

22.Jose S, Hamzah L, Jones R, Williams D, Winston A, Burns F, et al. Chronic Kidney Disease Risk in African and Caribbean Populations With HIV. J Infect Dis. 2018; 218(11):1767-72. https://doi.org/10.1093/ infdis/jiy397

23.Lopez ED, Córdova-Cázarez C, Valdez-Ortiz R, Cardona-Landeros CM, Gutiérrez-Rico MF. Fatores epidemiológicos, clínicos e laboratoriais associados à doença renal crônica em pacientes mexicanos infectados pelo HIV. J. Bras. Nefrol. 2019; 41(1): 48-54. https://doi.org/10.1590/2175-8239-jbn-2018-0024.

24.Kooij KW, Vogt L, Wit FWNM, Valk MV,van Zoest RA, Goorhuis A, et al. Higher Prevalence and Faster Progression of Chronic Kidney Disease in Human Immunodeficiency Virus-Infected Middle-Aged Individuals Compared With Human Immunodeficiency Virus-Uninfected Controls. J Infect Dis. 2017;216(6):622631. https://doi.org/10.1093/infdis/jix202.

25.Kirsztajn GM, Souza E, Romão Jr JE, Bastos MG, Meyer F, Andrada NC. Sociedade Brasileira de Nefrologia; Sociedade Brasileira de Urologia; Sociedade Brasileira de Pediatria; Sociedade Brasileira de Nutrição Parenteral e Enteral. Projeto Diretrizes Associação Médica Brasileira e Conselho Federal de Medicina. Doença Renal Crônica (Pré-terapia Renal Substitutiva): Diagnóstico. 2011 [citado em 06 sept 2019]. Disponível em: https://diretrizes.amb.org.br/_BibliotecaAntiga/doenca_renal_cronica_pre_terapia_renal_substitutiva_diagnostico.pdf

26.Webster AC, Nagler EV, Morton RL, Masson P. Chronic kidney disease. Lancet. 2017; 389:1238-52. https://doi.org/10.1016/ S0140-6736(16)32064-5

27.Dada SA, Olusegun OT, Ademola A, Adindu C, Olaitan RM, Oladimeji AA. A prevalência de doença renal crônica em pacientes recentemente diagnosticados com o vírus da imunodeficiência humana em llorin, Nigéria. J. Bras. Nefrol. 2015; 37(2):177-184. http://dx.doi.org/10.5935/0101-2800.20150029.

28.Brasil. Ministério da Saúde. Portaria no 1.675, de 7 de junho de 2018 [Internet]. 2018 [citado em 2019 set 25]. Disponível em: http://bvsms.saude.gov.br/bvs/saudelegis/gm/2018/prt1675_08_06_2018.html

29.Sales CB, Bernardes A, Gabriel CS, Brito MFP, Moura AA, Zanetti ACB. Protocolos Operacionais Padrão na prática profissional da enfermagem: utilização, fragilidades e potencialidades. Rev. Bras. Enferm. 2018; 71(1):126-134. http://dx.doi.org/10.1590/0034-7167-2016-0621. 